\title{
Neuroprotective actions of astaxanthin in cultured cortical neurones
}

\author{
D. Vauzour ${ }^{1,2}$, X. $\operatorname{Li}^{1}$, A. Bourquin ${ }^{1}$, X. Ou ${ }^{1}$, J. P. E. Spencer ${ }^{1}$ and P. Jauregi ${ }^{1}$ \\ ${ }^{1}$ Department of Food and Nutritional Sciences, School of Chemistry, Food and Pharmacy, University of Reading, \\ RG6 6AP, UK and ${ }^{2}$ Department of Nutrition, Institute of Biomedical and Clinical Science, Norwich Medical School, \\ University of East Anglia, Norwich NR4 7TJ, UK
}

\begin{abstract}
Abundant evidence exists to suggest that increased oxidative stress may contribute to the neuropathology of age-related brain disorders such as Alzheimer's and Parkinson's diseases ${ }^{(1,2)}$. Recently there has been an increased interest in the potential of dietary-derived phytochemicals to protect against neuronal damage associated with aging and neurodegenerative disorders. Astaxanthin, a natural carotenoid, is mainly used as a pigmentation source in aquaculture but increasing studies are proving its biological activity in protecting cell against oxidative stress with very interesting potential health applications such as in the treatment of degenerative diseases ${ }^{(3)}$. The major objective of this project was to investigate the ability of astaxanthin (synthetic $v$. natural) to protect against $\mathrm{H}_{2} \mathrm{O}_{2}$-induced neurotoxicity via its interactions with neuronal signalling pathways.
\end{abstract}

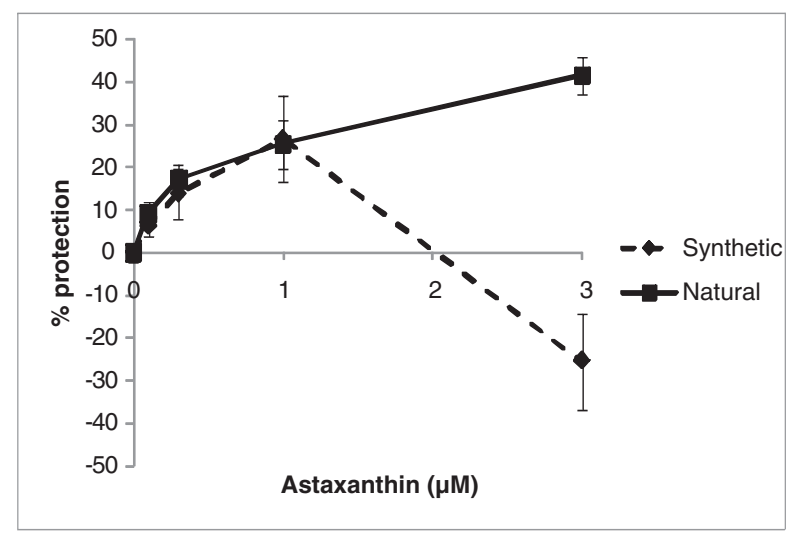

Fig. 1. Protective effect of astaxanthin against $\mathrm{H}_{2} \mathrm{O}_{2}$-induced neurotoxicity.

Both natural and synthetic astaxanthin did not induce neuronal injury as assessed by the Alamar blue assay $24 \mathrm{~h}$ post-exposure. Exposure of cortical neurons to $\mathrm{H}_{2} \mathrm{O}_{2}(75 \mu \mathrm{M}, 1 \mathrm{~h})$, resulted in a significant decrease in neuronal viability $(-48 \%, P<0.01)$. However, treatment with astaxanthin $(0.01-3 \mu \mathrm{M}, 18 \mathrm{~h})$ significantly protected neuronal cells against $\mathrm{H}_{2} \mathrm{O}_{2}$-induced toxicity. Interestingly the synthetic astaxanthin did not protect the neurons at the highest concentration of astaxanthin tested $(3 \mu \mathrm{M})$ while the natural astaxanthin showed a further increase in the protective effect (Fig. 1). The mechanism by which astaxanthin inhibited neuronal death was found to be linked to its ability to induce the activation of both Akt/PKB and the ERK1/2 pathways. Indeed, assessment of phospho-Akt revealed a bell-shape modulation with the greatest activation observed at $0.1 \mu \mathrm{M}$ and $0.3 \mu \mathrm{M}$ for natural and synthetic astaxanthin, respectively (1.3 fold increase, $P<0.05$ ). Interestingly, while natural astaxanthin exerted a dose-dependent activation in p-ERK1 (extracellular signalregulated kinases 1 ), synthetic astaxanthin differentially modulated p-EKR2 ( 2 fold increase, $P<0.05$ ). Such discrepancies may be due to differences in enantiomeric composition between the synthetic astaxanthin which is a mixture of four isomers (1:2(meso):1), and the natural which is produced as a pure enantiomer $\left(3 \mathrm{R}, 3^{\prime} \mathrm{R}\right)$ by the yeast Phaffia rhodozyma. The protective effects of astaxanthin against neurotoxins-induced toxicity will help shed light on their mechanism of neuroprotection.

1. Crossthwaite AJ, Hasan S \& Williams RJ (2002). Hydrogen peroxide-mediated phosphorylation of ERK1/2, Akt/PKB and JNK in cortical neurones: dependence on $\mathrm{Ca}(2+)$ and PI3-kinase. J Neurochem 80, 24-35.

2. Zhang Y, Dawson VL \& Dawson TM (2000). Oxidative stress and genetics in the pathogenesis of Parkinson's disease. Neurobiol Dis 7, 240-250.

3. Chang CH, Chen CY, Chiou JY et al. (2010) Astaxanthin secured apoptic death of PC12 cells induced by $\beta$-amyloid peptide. J Med Food 13, 548-556. 